\title{
Caracterización y comportamiento frente a la corrosión de recubrimientos de ácido fítico, obtenidos por conversión química, sobre substratos de magnesio en solución fisiológica
}

\author{
Laura A. Hernández-Alvarado ${ }^{\mathrm{a}}$, Martha A. Lomelí ${ }^{\mathrm{b}}$, Luis S. Hernández ${ }^{\mathrm{b}}$, Juana M. Miranda ${ }^{\mathrm{b}}$, \\ Lilia Narváez ${ }^{\mathrm{c}}$, Iván Díaz ${ }^{\mathrm{d}}$, María Cristina García-Alonso ${ }^{\mathrm{d}}$, María Lorenza Escudero ${ }^{\mathrm{d},}$ \\ ${ }^{a}$ Facultad de Ciencias Químicas, Universidad Autónoma de San Luis Potosí, Avda. \\ Dr. Manuel Nava 6, 78240 San Luis Potosí, S.L.P., México \\ ${ }^{b}$ Instituto de Metalurgia, Universidad Autónoma de San Luis Potosí, Avda. Dr. Manuel Nava 6, 78240 San Luis Potosí, SLP, México \\ ${ }^{\circ}$ Facultad del Hábitat, Universidad Autónoma de San Luis Potosí, Niño Artillero 150, 78290 | San Luis Potos, SLP, México \\ ${ }^{\mathrm{d}}$ Centro Nacional de Investigaciones Metalúrgicas (CENIM).CSIC. Avda. Gregorio del Amo 8, 28040 Madrid, España \\ Autor para la correspondencia: escudero@cenim.csic.es
}

\begin{abstract}
RESUMEN: Con objeto de mejorar la resistencia a la corrosión de implantes biodegradables de magnesio y de la aleación AZ31, se ha depositado un recubrimiento de ácido fítico sobre ambos sustratos y se ha evaluado su efecto protector frente a la corrosión. El recubrimiento fue obtenido mediante un tratamiento de conversión química, por inmersión en una solución de ácido fítico al $0,5 \%$ a $60^{\circ} \mathrm{C}$. Distintas técnicas (MEB/EDX, XRD y FTIR) fueron utilizadas para analizar la morfología del recubrimiento y su naturaleza química. Dichas técnicas mostraron que el recubrimiento de conversión era amorfo, con una composición química sobre la superficie de magnesio de $\mathrm{Mg}$, O y P y, además, Al, Zn y C sobre la superficie de la aleación AZ31. Los componentes principales del recubrimiento fueron quelatos formados por ácido fítico e iones metálicos. La resistencia a la corrosión de muestras sin recubrir y recubiertas, se evaluó mediante polarización potenciodinámica en solución de Hank a $37^{\circ} \mathrm{C}$. Los resultados indican que los recubrimientos de conversión de ácido fítico proporcionan una protección muy efectiva a los substratos de magnesio estudiados.
\end{abstract}

PALABRAS CLAVE: Ácido fítico; AZ31; Biomateriales; Corrosión; Magnesio; Recubrimientos de conversión química

Citation I Cómo citar este artículo: Hernández-Alvarado, L.A., Lomelí, M.A., Hernández, L.S., Miranda, J.M., Narváez, L., Díaz, I., García-Alonso, M.C., Escudero, M.L. (2014) "Caracterización y comportamiento frente a la corrosión de recubrimientos de ácido fítico, obtenidos por conversión química, sobre substratos de magnesio en solución fisiológica". Rev. Metal. 50(2): e012. doi: http://dx.doi.org/10.3989/revmetalm.012.

\begin{abstract}
Characterization and corrosion behavior of phytic acid coatings, obtained by chemical conversion on magnesium substrates in physiological solution. In order to improve the corrosion resistance of biodegradable magnesium and AZ31 magnesium alloy implants, a phytic acid coating has been applied on both substrates and their protective effect against corrosion has been assessed. The morphology and the chemical nature of the conversion coating were analyzed by SEM/EDX, XRD and FTIR. The spectra showed that the conversion coating was amorphous, and it was composed of $\mathrm{Mg}, \mathrm{O}$, and $\mathrm{P}$ on magnesium surface, along with $\mathrm{Al}, \mathrm{Zn}$ and $\mathrm{C}$ on AZ31 alloy. The main coating components were chelate compounds formed by phytic acid and metallic ions. The corrosion resistance of bare and coated samples was evaluated by potentiodynamic polarization technique in Hank's solution at $37^{\circ} \mathrm{C}$. The results indicate that phytic acid conversion coatings provided a very effective protection to the magnesium substrates studied.
\end{abstract}

KEYWORDS: AZ31; Biomaterials; Chemical conversion coating; Corrosion; Magnesium; Phytic acid

Copyright: $(\mathbb{C} 2014$ CSIC. This is an open-access article distributed under the terms of the Creative Commons AttributionNon Commercial (by-nc) Spain 3.0 License. 


\section{INTRODUCCIÓN}

El magnesio es uno de los elementos más abundantes en el cuerpo humano. Por ello, existe un elevado número de artículos que estudian su posible utilización como implante temporal, biodegradable y reabsorbible (Witte, 2010; Brar et al., 2011; Guo, 2011; Zheng y Gu, 2011; Kirkland et al., 2012). Si se compara con otros materiales metálicos implantables, el uso de materiales de base magnesio aporta una serie de ventajas, entre las que destacan: (a) un módulo elástico próximo al del hueso, bastante inferior al del acero inoxidable o titanio, (b) mayor tenacidad a la fractura que la hidroxiapatita, (c) mayor resistencia mecánica que los polímeros, (d) propiedades de biocompatibilidad y osteoactividad, y (e) permite la supresión de una segunda cirugía para retirar el implante cuando el hueso ha sanado. Además, el metabolismo del magnesio y su excreción en la orina es un proceso fisiológico que está bien entendido (Vormann, 2003). Dos son las principales cuestiones que se plantean a la hora de utilizar el magnesio y sus aleaciones como biomateriales temporales: (1) si la cinética de corrosión in vivo del magnesio y sus aleaciones es lo suficientemente lenta como para permitir que se repare el tejido dañado, y (2) qué elementos aleantes no presentan una dudosa biocompatibilidad.

La velocidad de corrosión del magnesio y sus aleaciones en el cuerpo humano debe ser controlada para evitar fenómenos inherentes a la misma, como, por ejemplo, la formación de hidrógeno gaseoso, que puede acumularse en tejidos adyacentes al implante e impedir el sanado. La liberación de hidrógeno puede conducir al desprendimiento mecánico de partículas de la aleación, designado como efecto de trozo (chunk effect). A estos problemas hay que añadir la necesidad de conseguir un método de ensayo in vitro que reproduzca las condiciones in vivo para una selección fiable y adecuada de las diferentes aleaciones de magnesio (Kirkland et al., 2010). La utilización de aleaciones de magnesio biodegradables como implantes médicos requiere un conocimiento extenso de su degradación en ambientes biológicos para asegurar su biocompatibilidad, integridad mecánica y, finalmente, la reabsorción y eliminación del material de implante sin producir efectos tóxicos no deseados (Álvarez-López et al., 2010; Carboneras et al., 2011).

Se ha señalado que el depósito de recubrimientos es una alternativa idónea para proteger al magnesio y sus aleaciones frente a la corrosión. De todos ellos, los recubrimientos de conversión química han recibido especial atención debido a su uniformidad, buena adherencia y coste reducido (Cui et al., 2008; Carboneras et al., 2010; Chen et al., 2011). Sin embargo, muchos factores pueden afectar a su calidad, de tal modo que si no se controla su proceso de depósito adecuadamente, pueden generar recubrimientos heterogéneos, con la presencia de poros, grietas u otros defectos que permitan la penetración del medio corrosivo hasta la superficie metálica. En consecuencia, uno de los grandes desafíos dentro del campo de los recubrimientos de conversión es producir recubrimientos uniformes, exentos de grietas, que aporten resistencia a la corrosión (Chen et al., 2011) y que, a la vez, sean biocompatibles y biodegradables.

Desde hace poco tiempo se ha utilizado ácido fítico (af) $\left(\mathrm{C}_{6} \mathrm{H}_{18} \mathrm{O}_{24} \mathrm{P}_{6}\right)$ para generar recubrimientos de conversión sobre aleaciones de magnesio (Jianrui et al., 2006; Cui et al., 2008; Gao et al., 2009; Pan et al., 2009). Cabe destacar, por ejemplo, su uso en los pretratamientos para recubrimientos electroless (Cui et al., 2010), en la oxidación por microarco (Zhang et al., 2010) o en la formación de capas autoensambladas (El-Sayed et al., 2012). Anteriormente, se ha empleado como inhibidor de la corrosión en pinturas y pigmentos (Notoya et al., 1995; Dost y Tokul, 2006), sin embargo, muy recientemente, se está explorando como recubrimiento para implantes médicos (Ye et al., 2012; Chen et al., 2013; Gupta et al., 2013). El af es un compuesto natural presente en la mayoría de las legumbres, granos de cereales y frijoles, representando, aproximadamente, el 70\% del contenido de fósforo en estos alimentos (Maga, 1982). La estructura peculiar del af hace que tenga una poderosa capacidad quelante con muchos iones metálicos. Si los grupos activos del af reaccionan con iones $\mathrm{Mg}^{2+}$ $\mathrm{o} \mathrm{Al}^{3+}$, formarían quelatos estables que podrían depositarse sobre la superficie de las aleaciones de magnesio, formando una capa de conversión química estable, con lo que aumentaría la resistencia a la corrosión (Jianrui et al., 2006; Cui et al., 2008). Por todo ello, el objetivo del presente trabajo ha sido generar un recubrimiento de ácido fítico sobre magnesio y la aleación AZ31, caracterizarlo química y morfológicamente y evaluar sus características protectoras frente a la corrosión en un medio fisiológico simulado.

\section{MATERIALES Y MÉTODOS}

\subsection{Substratos ensayados}

Los materiales usados como substratos fueron magnesio $(99,8 \%$ de pureza), obtenido por pulvimetalurgia y extruido, posteriormente, a $420{ }^{\circ} \mathrm{C}$ y la aleación AZ31, procesada mediante forja $(3,37 \%$ $\mathrm{Al} ; 0,78 \% \mathrm{Zn} ; 0,22 \% \mathrm{Mn}$ y resto $\mathrm{Mg}$ ). Las muestras de magnesio y AZ31 se embutieron en una resina acrílica de curado rápido, dejando una superficie expuesta al electrólito de $0,78 \mathrm{~cm}^{2}$ y $0,81 \mathrm{~cm}^{2}$, respectivamente. Previamente al depósito del recubrimiento, todas las muestras fueron desbastadas con papel de lija de $\mathrm{SiC}$ hasta grado 1200. 


\subsection{Recubrimiento aplicado}

El recubrimiento de ácido fítico fue depositado por inmersión en una solución de ácido fítico al $0,5 \%$, durante una hora y a $60{ }^{\circ} \mathrm{C}$ de temperatura (Jianrui et al., 2006). El pH de la solución se ajustó a 5,0 con trietilamina.

\subsection{Caracterización del recubrimiento}

\section{Morfológica}

La morfología del recubrimiento, tanto en superficie como en sección transversal, fue observada mediante Microscopía Electrónica de Barrido (MEB).

\section{Composicional}

El recubrimiento, aplicado sobre los diferentes substratos, fue caracterizado, composicionalmente, a través del sistema de microanálisis EDX incorporado en el microscopio electrónico de barrido, mediante espectroscopía infrarroja y difracción de rayos $\mathrm{X}$.

Para los análisis por espectroscopía infrarroja, el recubrimiento fue desprendido de los substratos con el uso de una navaja, se molió en polvo fino, se mezcló con $\mathrm{KBr}$ y se comprimió en una pastilla.

Por otro lado, el recubrimiento adherido a los substratos fue caracterizado mediante Difracción de Rayos X (DRX), empleando la técnica de haz paralelo en modo película (espejo Göbel), con un ángulo de incidencia de $2^{\circ}$. Se empleó la radiación $\mathrm{K} \alpha$ del cobre $(\lambda=1.54060 \AA)$, un voltaje de $40 \mathrm{kV}$, una intensidad de filamento de $40 \mathrm{~mA}$, un barrido angular de 4 a $80^{\circ}$, una anchura de paso de $0,02^{\circ}$ y un tiempo de adquisición por paso de 2 segundos.

\section{Electroquímica}

La evaluación de la resistencia a la corrosión de las muestras recubiertas, en comparación con los substratos desnudos, se llevó a cabo en solución de Hank (pH 7,8) a $37^{\circ} \mathrm{C}$, cuya composición, en $\mathrm{g} \mathrm{L}^{-1}$, corresponde a: $\mathrm{NaCl} 8,0 ; \mathrm{CaCl}_{2} 0,14 ; \mathrm{KCl} \mathrm{0,40;}$ $\mathrm{MgCl}_{2} \cdot 6 \mathrm{H}_{2} \mathrm{O} \quad 0,10 ; \mathrm{MgSO}_{4} \cdot 7 \mathrm{H}_{2} \mathrm{O} \quad 0,06 ; \mathrm{NaHCO}_{3}$ 0,$35 ; \mathrm{Na}_{2} \mathrm{H}_{2} \mathrm{PO}_{4} 0,10 ; \mathrm{Na}_{2} \mathrm{HPO}_{4} \cdot 2 \mathrm{H}_{2} \mathrm{O} 0,06$; glucosa 1,00 .

Se realizaron curvas de polarización potenciodinámica después de una hora de estabilización del potencial a circuito abierto $\left(\mathrm{E}_{\mathrm{ca}}\right)$. El barrido comenzó en $-500 \mathrm{mV}$ hasta alcanzar $+1000 \mathrm{mV}$, ambos respecto a $E_{c a}$, y a una velocidad de barrido de $1 \mathrm{mV} \mathrm{s}^{-1}$. Se utilizó un electrodo de referencia de calomelanos saturado (ecs) y dos barras de grafito de alta densidad como electrodos auxiliares. La reproducibilidad se confirmó ensayando un mínimo de 2 muestras idénticas por cada condición superficial.

\section{RESULTADOS Y DISCUSIÓN}

\subsection{Morfología del recubrimiento}

La observación de la superficie del recubrimiento generado en la aleación AZ31 revela numerosas cavidades (Fig. 1a). En algunas partes, el recubrimiento está fracturado y/o desprendido, observándose por debajo una capa similar. Aparentemente el recubrimiento es de pequeño espesor, ya que creció siguiendo las rayas del lijado, aún visibles. Dos son las causas que pueden conducir a la formación de las cavidades: la primera, un tiempo muy prolongado al aire antes del lavado, lo que pudo ocasionar que se secara la solución remanente sobre la superficie (Hillis, 1994); y la segunda, la ausencia de agitación durante la formación del recubrimiento.

El recubrimiento de af sobre la superficie de magnesio (Fig. 1b) revela que está constituido por dos estratos, uno superior, desprendido en muchas zonas, y otro inferior, aparentemente continuo pero agrietado. Las microfisuras se crean muy probablemente, bien por el desprendimiento de hidrógeno durante la reacción o bien por la deshidratación y contracción durante su secado.

\subsection{Composición del recubrimiento}

\section{Microscopía electrónica de barrido (MEB/EDX)}

El resultado del análisis por dispersión de energías de rayos X (EDX) de la Figura 2, muestra la presencia de $\mathrm{C}, \mathrm{O}, \mathrm{Mg}, \mathrm{Al}$ y $\mathrm{P}$ en el recubrimiento generado sobre la aleación AZ31, y de $\mathrm{O}, \mathrm{Mg}$ y $\mathrm{P}$ en el recubrimiento sobre magnesio. De estos elementos, el $\mathrm{Mg}$ y el Al provienen de los substratos metálicos, $\mathrm{y}$ el resto del $a f$. La presencia de $\mathrm{P}$ indica que el recubrimiento de conversión de af recubre la superficie del substrato.

\section{Espectroscopía infrarroja}

La Figura 3 presenta espectros de infrarrojo del ácido fítico y del recubrimiento de conversión con ácido fítico, que es representativo para ambos sustratos metálicos (Mg y AZ31). Para el primero, las bandas características se centran a 3500-3000, 1630, 1000 y $500 \mathrm{~cm}^{-1}$, que corresponden a $\mathrm{OH}^{-}, \mathrm{HPO}_{4}{ }^{2-}$, $\mathrm{PO}_{4}{ }^{3-}{\mathrm{y} \mathrm{PO}_{4}}^{3-}$, respectivamente; mientras que para el espectro del recubrimiento de conversión de af, la presencia de bandas a 3500-3000 y $1630 \mathrm{~cm}^{-1}$ demuestra la existencia de oxidrilos en el recubrimiento. Comparando ambos espectros, se aprecia que las bandas del recubrimiento se desplazan ligeramente a mayores números de onda con respecto a las bandas del $a f$. El desplazamiento de la banda situada a $1000 \mathrm{~cm}^{-1}$ y la degradación de la banda a $500 \mathrm{~cm}^{-1}$, indican que el af ha formado compuestos quelantes con los iones metálicos, habiéndose 

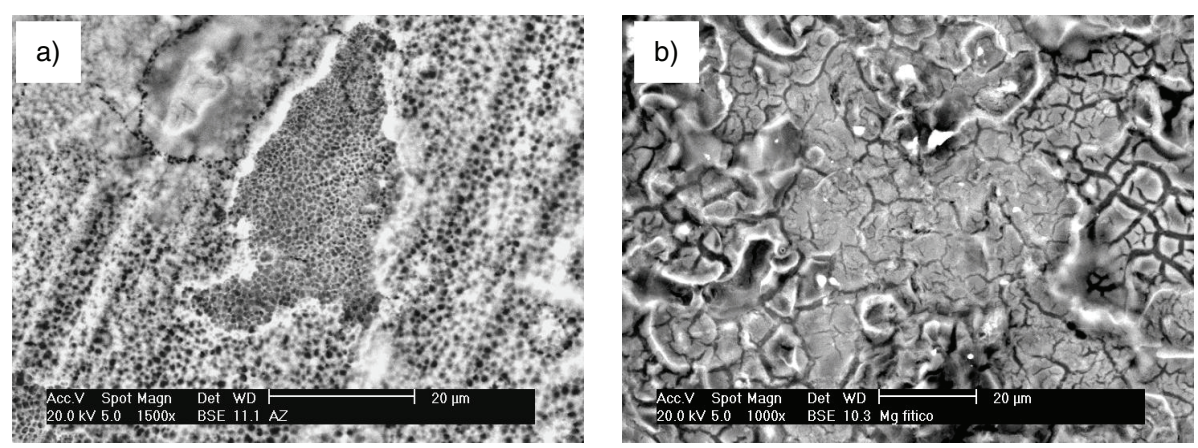

FIgURA 1. Aspecto de los recubrimientos de conversión de ácido fítico: a) sobre la aleación AZ31 y b) sobre magnesio pulvimetalúrgico.

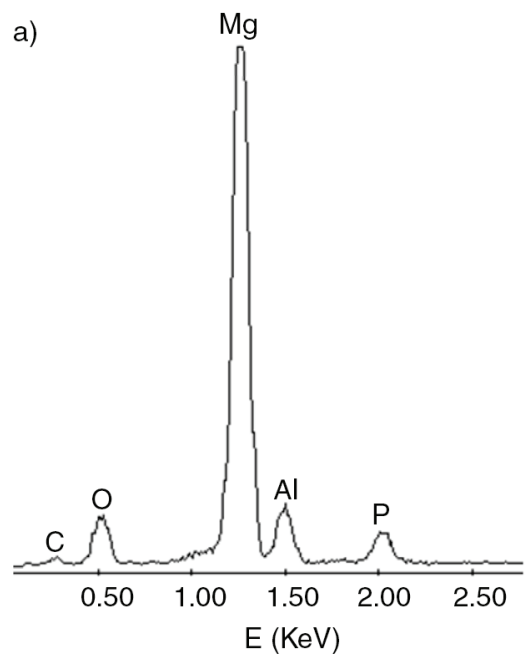

b)

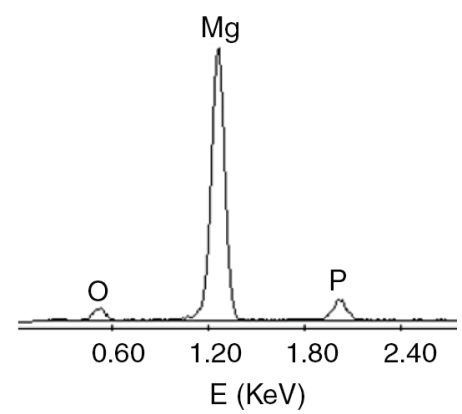

Figura 2. Análisis por espectroscopía de energías dispersadas de rayos X (EDX) del recubrimiento de ácido fítico sobre la aleación AZ31 (a) y sobre magnesio (b).

depositado posteriormente sobre el substrato (Gao et al., 2009; Pan et al., 2009). Los productos de la reacción fueron adsorbidos sobre la superficie del substrato. Por tanto, considerando los resultados de infrarrojo y los del análisis por EDX (Fig. 2), puede concluirse que el recubrimiento de conversión es el producto de reacción del af con las superficies de magnesio y de la aleación AZ31.

\section{Difracción de rayos X (DRX)}

La Figura 4 muestra un difractograma obtenido mediante DRX, representativo del recubrimiento de af depositado sobre la aleación AZ31. Los máximos de difracción más agudos corresponden al magnesio existente en los substratos metálicos (PDF 00-001-1141, International Center for Diffraction Data, 2010), mientras que el resto pertenece al recubrimiento de $a f$. Sin embargo, únicamente, ha sido posible identificar al compuesto $\mathrm{AlPO}_{4}(\mathrm{PDF}$
01-070-6291, International Center for Diffraction Data, 2010), cuyo máximo de difracción se encuentra situado a $7,54^{\circ}$. La naturaleza amorfa del recubrimiento impide la asignación inequívoca del máximo de difracción restante que aparece a bajos ángulos. Similares resultados se obtienen para muestras de magnesio recubierto con $a f$.

\section{Comportamiento electroquímico}

La Figura 5a muestra la evolución del potencial a circuito abierto $\left(E_{c a}\right)$ de los substratos de magnesio y AZ31 durante la formación del recubrimiento de conversión por inmersión en la solución de af a $60{ }^{\circ} \mathrm{C}$. El $\mathrm{E}_{\mathrm{ca}}$ se ennoblece durante los primeros 300 segundos de inmersión. El incremento abrupto se debe, probablemente, a la activación de la superficie y al crecimiento del recubrimiento. Después de unos 600 segundos, el $\mathrm{E}_{\mathrm{ca}}$ tiende a estabilizarse, lo que indica que el proceso de conversión ha finalizado. 


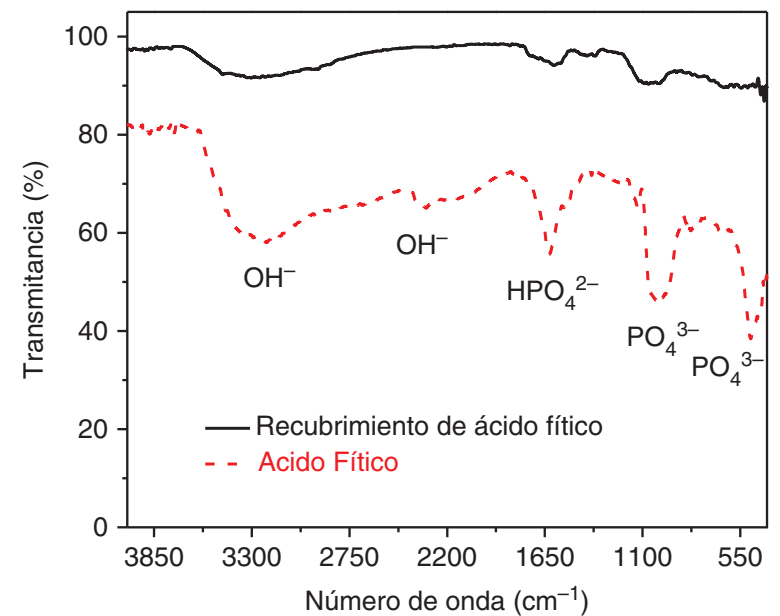

Figura 3. Espectros de infrarrojo del ácido fítico y del recubrimiento de conversión con ácido fítico, representativo de ambos sustratos metálicos (magnesio y aleación AZ31).

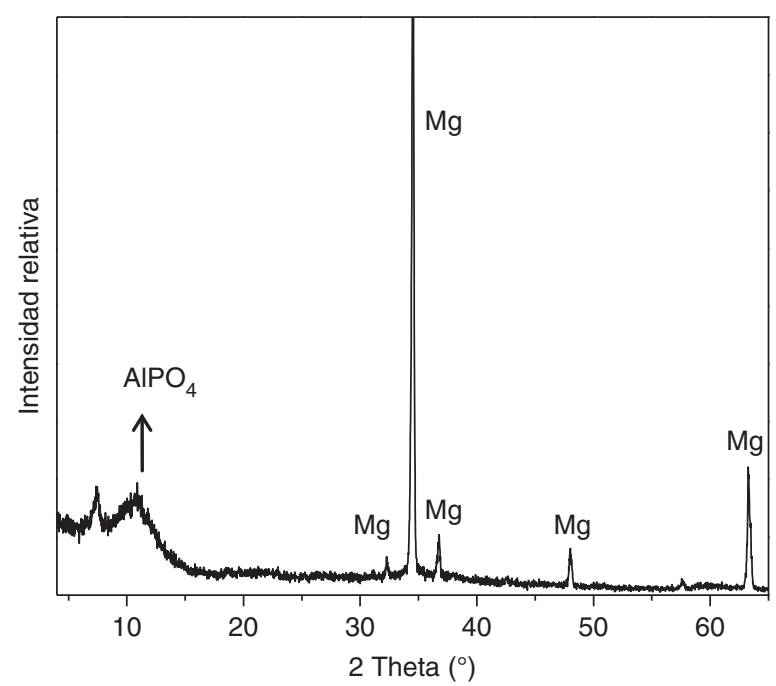

Figura 4. Espectro de difracción de rayos X representativo de la aleación AZ31, recubierta con la solución de ácido fítico.

Las muestras de la aleación AZ31 y de magnesio, no recubiertas y con la presencia del recubrimiento de $a f$, fueron evaluadas cualitativamente mediante el seguimiento del $E_{c a}$ en función del tiempo de inmersión en solución de Hank a $37^{\circ} \mathrm{C}$ (ver Fig. 5b). En las muestras recubiertas con $a f$, se observa un desplazamiento del $\mathrm{E}_{\mathrm{ca}}$ hacia potenciales más nobles, adquiriendo un valor de potencial de unos $-150 \mathrm{mV}$ transcurridos 3600 segundos. Este comportamiento es semejante al de una aleación cuya capa de pasivación permanece intacta después de un periodo en el que engrosa y corrige sus imperfecciones (González, 1989). Las muestras sin recubrir exhibieron un comportamiento monótono, con potenciales muy negativos (en torno a $-1700 \mathrm{mV}$ ), durante todo el ensayo.
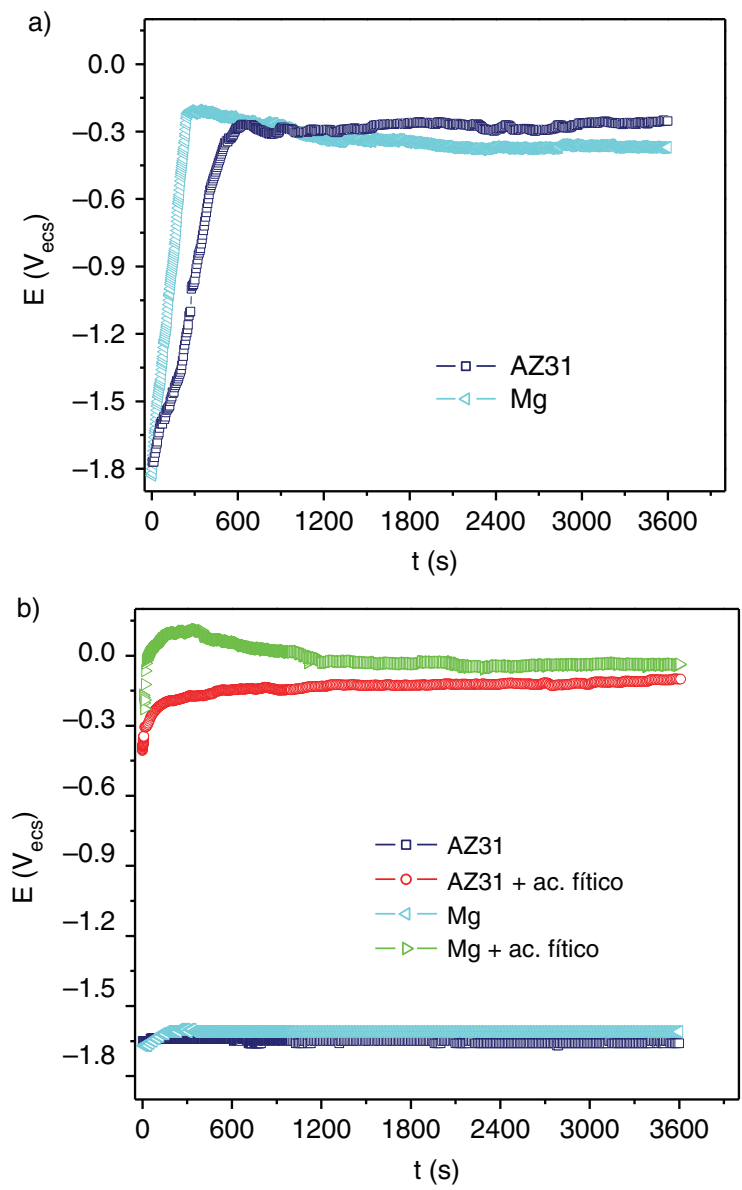

FigurA 5. (a) Evolución del $\mathrm{E}_{\mathrm{ca}}$ de la aleación AZ31 y del magnesio, durante la formación del recubrimiento por tratamiento de conversión en ácido fítico y (b) comparación de la evolución del $\mathrm{E}_{\mathrm{c}}$ de la aleación AZ31 y del magnesio, sin recubrir y recubiertos con ácido fítico en solución de Hank.

En la Figura 6, se muestran las curvas de polarización potenciodinámica para los substratos desnudos de magnesio y AZ31, y recubiertos con fítico en solución de Hank. Los valores del $\mathrm{E}_{\mathrm{ca}}$, densidad de corriente $\left(\mathrm{i}_{\text {corr }}\right)$ y las pendientes anódica $\left(\beta_{a}\right)$ y catódica $\left(\beta_{c}\right)$ procedentes de las curvas de polarización, se resumen en la Tabla 1 . Se puede apreciar que la presencia del recubrimiento desplaza el $E_{c a}$ hacia valores mucho más nobles con respecto a las muestras con ausencia del mismo (Tabla 1 y Figuras 5 y 6 ). También se puede apreciar que los recubrimientos originan curvas de polarización potenciodinámica claramente desplazadas hacia menores densidades de corriente, en torno a 2 ó 3 órdenes de magnitud inferiores con respecto a las muestras no recubiertas. Estos resultados son semejantes a los observados en aleaciones AZ91 y $\mathrm{Mg}$ - $\mathrm{Li}$, ensayadas en $\mathrm{NaCl}$ al 3,5\% a temperatura ambiente (Cui et al., 2008; Gao et al., 2009). El control del proceso corrosivo en los sustratos desnudos es catódico, mientras que los mismos materiales recubiertos con af presentan 


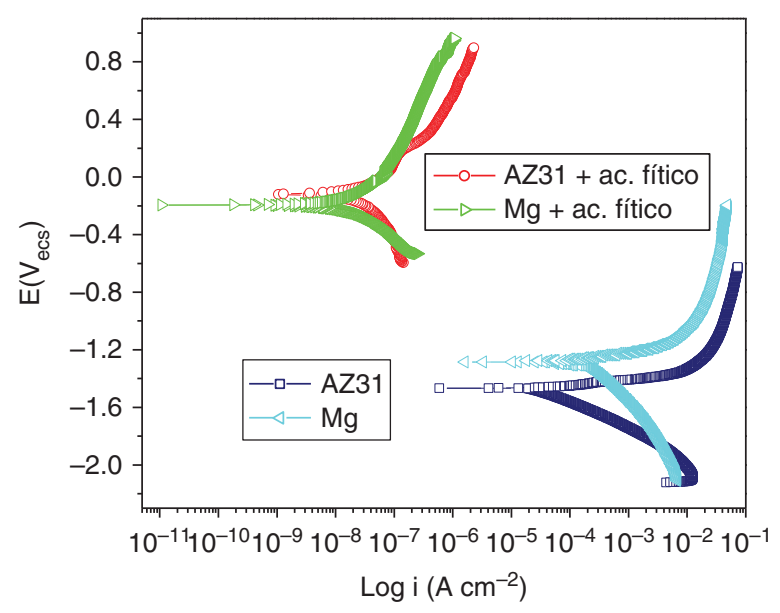

Figura 6. Curvas de polarización potenciodinámica de la aleación AZ31 y del magnesio, sin recubrir y recubiertos con ácido fítico, en la solución de Hank.

un control anódico de la corrosión (ver Tabla 1). Las curvas para las muestras recubiertas son prácticamente coincidentes, indicando que su comportamiento es muy semejante. Sin embargo, no presentan un rango de pasividad claro, contrariamente a lo que se ha informado que sucede cuando el medio es el tampón fosfato salino (PBS), ensayado a temperatura ambiente (Hernández et al., 2010). No obstante, tampoco se aprecia un aumento brusco de la corriente, que indique una rotura del recubrimiento.

Generalmente, en los trabajos relativos a recubrimientos de conversión de af sobre aleaciones de magnesio, se resaltan al menos dos aspectos: primero, los recubrimientos se ensayan, solamente, en soluciones de $\mathrm{NaCl}$ a temperatura ambiente y a tiempos cortos de exposición y segundo, no se suele proporcionar evidencia gráfica sobre su espesor, a pesar de los buenos resultados obtenidos. Uno de los objetivos que propiciaron el presente estudio, fue verificar si se obtendrían resultados aceptables de esta misma combinación de recubrimiento y substratos de magnesio, en una solución fisiológica simuladas a $37^{\circ} \mathrm{C}$. Es preciso reseñar que el af se degrada con la temperatura, ya que al hidrolizarse se pueden generar derivados con menor número de fosfatos e incluso inositol libre, con menor capacidad de unirse a iones metálicos (http://agendaquimica.blogspot. $\mathrm{mx} / 2011 / 12 /$ el-acido-fitico-y-los-fitatos.html). En relación al mecanismo del proceso de quelación con af sobre una aleación de magnesio, se ha propuesto lo siguiente (Dost y Tokul, 2006; Cui et al., 2008): El af tiene 12 grupos oxidrilo y 6 grupos fosfato, lo que le confiere una gran capacidad de quelación con muchos iones metálicos en un amplio intervalo de $\mathrm{pH}$. En soluciones acuosas, el af se ioniza, en función del pH, para formar el ión hidronio, el ión del af con diferente número de iones fosfato y el ión $\mathrm{HPO}_{4}{ }^{2-}$. Al mismo tiempo, un gran número de iones $\mathrm{Mg}^{2+}$ son generados cuando la aleación de magnesio se sumerge en la solución de af. Posteriormente, estos iones se combinan con los iones del af para formar complejos fitato fítico-Mg, que se depositan sobre la superficie del substrato. Se acepta que los iones metálicos formadores de quelatos son $\mathrm{Mg}^{2+} \mathrm{y}$ $\mathrm{Al}^{3+}$, pero podrían participar también los iones del Zn y Mn, por ejemplo, aunque estos elementos usualmente no se detectan por EDX. A partir de resultados de EDX, se ha señalado que la formación de recubrimientos de conversión de af sobre una aleación AZ (Pan et al., 2009), debe atribuirse a quelatos de $\mathrm{Al}$ insolubles, que precipitan sobre el substrato, pero no a los quelatos solubles del resto de elementos metálicos. En el presente estudio, no obstante, se obtuvieron los recubrimientos de conversión protectores sobre magnesio puro, lo que contradice la afirmación anterior.

Respecto al espesor del recubrimiento de conversión, la Figura 7 presenta un corte de sección transversal del recubrimiento depositado sobre la aleación AZ31. La superficie aparece compacta y continua, pero debajo de ella se aprecian cavidades alargadas que conectan con el substrato y que deben corresponder a las grietas mostradas en la Figura 1. Una morfología semejante se observó en el recubrimiento generado sobre magnesio. Por tanto, surge la pregunta de si después de 24 horas de exposición en el electrolito no se ha disuelto completamente la zona continua, o si ocurre un proceso de sellado de las grietas y cavidades. En la mayoría de los trabajos revisados aparecen grietas en la superficie del recubrimiento y la mayor resistencia a la corrosión parece coincidir con la red más fina de grietas.

TABla 1. Parámetros de corrosión de magnesio y de la aleación AZ31, desnudos y recubiertos con ácido fítico, obtenidos en los ensayos de polarización potenciodinámica en solución de Hank

\begin{tabular}{|c|c|c|c|c|c|}
\hline Material metálico & $\begin{array}{c}\mathrm{E}_{\mathrm{ca}} \\
\left(\mathrm{mV_{ \text {ecs } }}\right)\end{array}$ & $\left(\mu \mathrm{A}^{\mathbf{c}_{\text {corr }}} \mathrm{cm}^{-2}\right)$ & $\begin{array}{l}\text { Velocidad de corrosión } \\
\qquad(\mu \mathrm{m} / \mathbf{a n ̃ o})\end{array}$ & $\begin{array}{c}\beta_{\text {anódica }} \\
\text { (mV/decada) }\end{array}$ & $\begin{array}{c}\beta_{\text {catódica }} \\
\text { (mV/decada) }\end{array}$ \\
\hline $\mathrm{Mg}$ & -1316 & 148 & 3404 & 41 & 281 \\
\hline AZ31 & -1520 & 17 & 711 & 40 & 176 \\
\hline $\mathrm{Mg}+$ ac. fítico & -206 & 0,04 & 1,02 & 864 & 496 \\
\hline AZ31 + ac. fítico & -183 & 0,12 & 2,79 & 575 & 66 \\
\hline
\end{tabular}




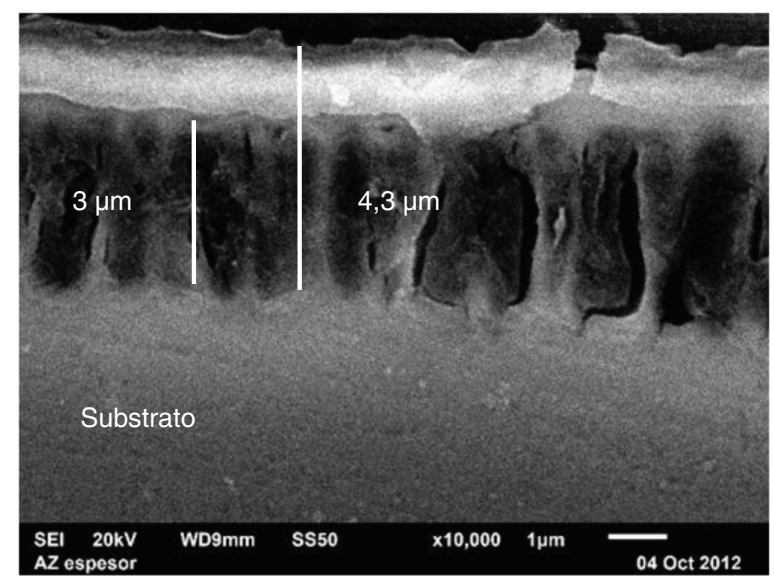

Figura 7. Sección transversal del recubrimiento de ácido fítico sobre la aleación AZ31.

\section{CONCLUSIONES}

Los resultados de los ensayos electroquímicos demuestran que la resistencia a la corrosión del magnesio pulvimetalúrgico y la aleación AZ31 puede incrementarse, considerablemente, en solución de Hank a $37^{\circ} \mathrm{C}$, creando un recubrimiento de conversión de ácido fítico mediante una simple inmersión. Las curvas de polarización potenciodinámica indican una mayor resistencia a la corrosión de los sustratos de magnesio y AZ31 tratadas con ácido fítico. Mediante las técnicas de análisis superficial y caracterización del recubrimiento en estudio, se puede concluir que los quelatos se forman por la reacción entre los grupos activos del ácido fítico y los iones metálicos. Esta capa de conversión química protege efectivamente las superficies de magnesio y AZ31 del medio corrosivo.

\section{AGRADECIMIENTOS}

Los autores agradecen la ayuda a Antonio Alberto Torres y Claudia Hernández, por la obtención de las imágenes del recubrimiento en sección transversal, así como su ayuda en la realización de los ensayos de difracción de rayos X. Por último, los autores agradecen la financiación recibida de los proyectos del Plan Nacional MAT 2008-06719-C0301 and MAT 2011-29152-C02-01.

\section{REFERENCIAS}

Álvarez-López, M., Pereda, M.D., del Valle, J.A., FernándezLorenzo, M., García-Alonso, M.C., Ruano, O.A., Escudero, M.L. (2010). Corrosion behaviour of AZ31 magnesium alloy with different grain sizes in simulated biological fluids. Acta Biomater. 6(5), 1763-1771. http://dx.doi.org/10.1016/j. actbio.2009.04.041.

Brar, H.S., Keselowsky, B.G., Sarntinoranont, M., Manuel, M.V. (2011). Design considerations for developing biodegradable and bioresorbable magnesium implantes. JOM 63 (4), 100104. http://dx.doi.org/10.1007/s11837-011-0048-8.

Carboneras, M., Hernández-Alvarado, L.A., Mireles, I.E., Hernández, L.S., García-Alonso, M.C., Escudero, M.L. (2010). Tratamientos químicos de conversión para la protección de magnesio biodegradable en aplicaciones temporales de reparación ósea. Rev. Metal. 46 (1), 86-92. http:// dx.doi.org/10.3989/revmetalm.0944

Carboneras, M., Iglesias, C., Pérez-Maceda, B.T., del Valle, J.A., García-Alonso, M.C., Alobera, M.A., Clemente, C., Rubio, J.C., Escudero, M.L., Lozano, R.M. (2011). Comportamiento frente a la corrosión y biocompatibilidad in vitro/in vivo de la aleación AZ31 modificada superficialmente. Rev. Metal. 47 (3), 212-223. http://dx.doi.org/10.3989/revmetalm.1065.

Chen, X.B., Birbilis, N., Abbott, T.B. (2011). Review of corrosionresistant conversion coatings for magnesium and its alloys. Corrosion 67 (3), 035005-1-035005-16. http://dx.doi.org/ 10.5006/1.3563639.

Chen, Y., Wan, G., Wang, J., Zhao, S., Zhao, Y., Huang, N. (2013). Covalent immobilization of phytic acid on $\mathrm{Mg}$ by alkaline pre-treatment: Corrosion and degradation behavior in phosphate buffered saline. Corros. Sci. 75, 280-286. http://dx.doi. org/10.1016/j.corsci.2013.06.011.

Cui, X., Li, Q., Li, Y., Wang, F., Jin, G., Ding, M. (2008). Microstructure and corrosion resistance of phytic acid conversion coatings for magnesium alloy. Appl. Surf. Sci. 255 (5) Part 1, 2098-2103. http://dx.doi.org/10.1016/j.apsusc.2008.06.199.

Cui, X., Jin, G., Li, Q., Yang, Y., Li, Y., Wang, F. (2010). Electroless Ni-P plating with a phytic acid pretreatment on AZ91D magnesium alloy. Mat. Chem. Phys. 121 (1-2), 308-313. http://dx.doi.org/10.1016/j.matchemphys.2010.01.042.

Dost, K., Tokul, O. (2006). Determination of phytic acid in wheat and wheat products by reverse phase high performance liquid chromatography. Anal. Chim. Acta 558 (1-2), 22-27. http://dx.doi.org/10.1016/j.aca.2005.11.035.

El-Sayed, A-R., Harm, U., Mangold, K-M., Fürbeth, W. (2012). Protection of galvanized steel from corrosion in $\mathrm{NaCl}$ solution by coverage with phytic acid SAM modified with some cations and thiols. Corros. Sci. 55, 339-350. http://dx.doi. org/10.1016/j.corsci.2011.10.036.

Gao, L., Zhang, C., Zhang, M., Huang, X., Jiang, X. (2009). Phytic acid conversion coating on Mg-Li alloy. J. Alloys compd. 485 (1-2), 789-793. http://dx.doi.org/10.1016/j. jallcom.2009.06.089.

González, J.A. (1989). Control de la corrosión. Estudio y medida por técnicas electroquímicas. CSIC, Madrid (España).

Guo, K.W. (2011). A review of magnesium/magnesium alloys corrosion. Recent Pat. Corros. Sci. 1, 72-90.

Gupta, R.K., Mensah-Darkwa, K., Kumar, D. (2013). Effect of post heat treatment on corrosion resistance of phytic acid conversion coated magnesium. J. Mater. Sci. Technol. 29 (2), 180-186. http://dx.doi.org/10.1016/j.jmst.2012.12.014.

Hernández L.A., Hernández, L.S., Hernández, G., Escudero, M.L. (2010). Artículo 079. Comunicación presentada en el XXV Congreso Nacional de la Sociedad Mexicana de Electroquímica, Zacatecas, México.

Hillis, J.E. (1994). Surface Engineering of magnesium alloys en ASM Handbook, Surface engineering, Vol 5, ASM international, pp. 819-834.

Jianrui, L., Yina, G., Weidong, H. (2006). Study on the corrosion resistance of phytic acid conversion coating for magnesium alloys. Surf. Coat. Technol. 201, 1536-1541. http://dx.doi. org/10.1016/j.surfcoat.2006.02.020.

Kirkland, N.T., Lespagnol, J., Birbilis, N., Staiger, M.P. (2010). A survey of bio-corrosion rates of magnesium alloys. Corros. Sci. 52, 287-291. http://dx.doi.org/10.1016/j.corsci. 2009.09.033.

Kirkland, N.T., Birbilis, N., Staiger, M.P. (2012). Assessing the corrosion of biodegradable magnesium implants: A critical review of current methodologies and their limitations. Acta Biomater. 8 (3), 925-936. http://dx.doi.org/10.1016/j. actbio.2011.11.014.

Maga, J.A. (1982). Phytate: its chemistry, occurrence, food interactions, nutritional significance, and methods of analysis. $J$. Agric. Fodd Chem. 30 (1), 1-9. http://dx.doi.org/10.1021/jf $00109 \mathrm{a} 001$. 
Notoya, T., Otieno-Alego, V., Schweinsbert, D.P. (1995). The corrosión and polarization behaviour of copper in domestic wáter in the presence of $\mathrm{Ca}, \mathrm{Mg}$, and $\mathrm{Na}$-salts of phytic acid. Corros. Sci. 37 (1), 55-65. http://dx.doi.org/ 10.1016/0010-938X(94)00105-F

Pan, F., Yang, X., Zhang, D. (2009). Chemical nature of phytic acid conversion coating on AZ61 magnesium alloy. Appl. Surf. Sci. 255, 8363-8371. http://dx.doi.org/10.1016/j.apsusc. 2009.05.089.

Vormann, J. (2003). Magnesium: nutrition and metabolism. Mol. Aspects Med. 24 (1-3), 27-37. http://dx.doi.org/10.1016/ S0098-2997(02)00089-4.

Witte, F. (2010). The history of biodegradable magnesium implants: A review. Acta Biomater. 6 (5), 1680-1692. http:// dx.doi.org//10.1016/j.actbio.2010.02.028.
Ye, C.H., Zheng, Y.F., Wang, S.Q., Xi, T.F., Li, Y.D. (2012). In vitro corrosión and biocompatibility sudy of phytic acid modified WE43 magnesium alloy. Appl. Surf. Sci. 258 (8), 3420-3427. http://dx.doi.org/10.1016/j.apsusc.2011. 11.087

Zhang, R.F., Xiong, G.Y., Hua, C.Y. (2010). Comparison of coating properties obtained by MAO on magnesium alloys in silicate and phytic acid electrolytes. Curr. Appl. Phys. 10 (1), 255-259. http://dx.doi.org/10.1016/j.cap.2009. 06.008 .

Zheng, Y., Gu, X. (2011). Research activities of biomedical magnesium alloys in China. JOM 63 (4), 105-108. http:// dx.doi.org/10.1007/s11837-011-0049-7. 